\title{
Rilonacept maintains long-term inflammatory remission in patients with deficiency of the IL-1 receptor antagonist
}

\author{
Megha Garg, ${ }^{1}$ Adriana A. de Jesus, ${ }^{1}$ Dawn Chapelle, ${ }^{2}$ Paul Dancey, ${ }^{3}$ Ronit Herzog, ${ }^{4}$ \\ Rafael Rivas-Chacon, ${ }^{5}$ Theresa L. Wampler Muskardin, ${ }^{6}$ Ann Reed, ${ }^{7}$ James C. Reynolds, ${ }^{8}$ \\ Raphaela Goldbach-Mansky, ${ }^{1}$ and Gina A. Montealegre Sanchez ${ }^{1}$ \\ 'Translational Autoinflammatory Disease Studies, National Institute of Allergy and Infectious Diseases (NIAID), NIH, \\ Bethesda, Maryland, USA. ${ }^{2}$ National Institute of Arthritis and Musculoskeletal and Skin Diseases (NIAMS), NIH, Bethesda, \\ Maryland, USA. ${ }^{3}$ Memorial University of Newfoundland, St. John's, Newfoundland, Canada. ${ }^{4}$ New York University, New \\ York, New York, USA. ${ }^{5}$ Miami Children's Hospital, Miami, Florida, USA. ${ }^{6}$ Mayo Clinic Rochester, Rochester, Minnesota, USA \\ ${ }^{7}$ Duke University School of Medicine, Durham, North Carolina, USA. ${ }^{8}$ Radiology and Imaging Sciences, Clinical Center, NIH, \\ Bethesda, Maryland, USA.
}

BACKGROUND. Deficiency of IL-1 receptor antagonist (DIRA) is a rare autoinflammatory disease that presents with life-threatening systemic inflammation, aseptic multifocal osteomyelitis, and pustulosis responsive to IL-1-blocking treatment. This study was performed (a) to investigate rilonacept, a long-acting IL-1 inhibitor, in maintaining anakinra-induced inflammatory remission in DIRA patients, (b) to determine doses needed to maintain remission, and (c) to evaluate the safety and pharmacokinetics of rilonacept in young children ( $<12$ years).

METHODS. Six mutation-positive DIRA patients (children, ages 3-6 years), treated with daily anakinra, were enrolled into an open-label pilot study of subcutaneous rilonacept for 24 months. Clinical symptoms and inflammatory blood parameters were measured at all visits. A loading dose ( $4.4 \mathrm{mg} / \mathrm{kg}$ ) was administered, followed by once weekly injections $(2.2 \mathrm{mg} / \mathrm{kg}$ ) for 12 months. Dose escalation $(4.4 \mathrm{mg} / \mathrm{kg}$ ) was allowed if inflammatory remission was not maintained. Subjects in remission at 12 months continued rilonacept for an additional 12 months.

RESULTS. Five of six patients required dose escalation for findings of micropustules. Following dose escalation, all patients were in remission on weekly rilonacept administration, with stable laboratory parameters for the entire study period of $\mathbf{2 4}$ months. All children are growing at normal rates and have normal heights and weights. Quality of life improved while on rilonacept. No serious adverse events were reported.

CONCLUSION. Rilonacept was found to maintain inflammatory remission in DIRA patients. The once weekly injection was well tolerated and correlated with increased quality of life, most likely related to the lack of daily injections.

Conflict of interest: R. GoldbachMansky has received study support under a Cooperative Research and Development Agreement from Regeneron, SOBI, Novartis, and E. Lilly. C.A. Montealegre Sanchez has received study support under a Cooperative Research and Development Agreement from Regeneron and E. Lilly.

Submitted: May 3, 2017 Accepted: July 11, 2017 Published: August 17, 2017

\section{Reference information:} JCI Insight. 2017;2(16):e94838. https://doi.org/10.1172/jci. insight. 94838.
TRIAL REGISTRATION. ClinicalTrials.gov NCT01801449.

FUNDING. NIH, NIAMS, and NIAID.

\section{Introduction}

Deficiency of IL-1 receptor antagonist (DIRA) is a monogenic autoinflammatory disease caused by loss of function mutations in the gene encoding IL-1 receptor antagonist (IL-1Ra) that result in unopposed signaling of the proinflammatory cytokines IL- $1 \alpha$ and IL-1 $\beta$ through the IL- 1 receptor. DIRA presents with multifocal osteomyelitis and pustulosis and can escalate to life-threatening inflammation, with the development of systemic inflammatory response syndrome (SIRS) and organ failure $(1,2)$. DIRA patients are homozygous or compound heterozygous for loss-of-function mutations in $I L 1 R N$, encoding IL-1Ra. Most mutations are nonsense or frameshift mutations that lead to either no expression of protein $(2,3)$ or expression of nonfunctional protein (4). To date, 10 different disease-causing mutations in $I L 1 R N$ have been found, including 4 
nonsense mutations, 1 in-frame deletion, 3 frameshift deletions, and a 22-kb and a genomic 175-kb deletion on chromosome 2 (2-6). The 175-kb deletion seen in the DIRA patients of Puerto Rican descent encompasses $I L 1 R N$ as well as 5 adjacent genes, including antagonist $I L 36 R N$; agonists $I L 36 A, I L 36 B$, and $I L 36 G$ of the IL-36 receptor; and $\operatorname{IL38}(2,3,7)$.

Mortality is difficult to estimate but is approximately $30 \%$ in early infancy. Reports of mortality in cases from regions, where disease-causing, likely founder mutations have been identified, in retrospect, are likely to have been DIRA (8). DIRA can cause fetal demise in utero. A postmortem histopathological evaluation of a 27-week fetus (9) showed dense neutrophilic infiltrates, with tissue destruction in the thymus, adrenal gland, and myocardium, and a postmortem bone x-ray suggested osteomyelitis. The consanguineous parents had a second child who was born at 31 weeks gestational age and died at the age of 4 months with skin pustulosis, multifocal osteomyelitis, and increased inflammatory markers. There was no DNA available from either patient, but their parents were found to be heterozygous for a likely disease-causing nonsense mutation in $I L 1 R N$. These findings point to a role of IL-1Ra in tissue protection during fetal development. To our knowledge, there are no patients with DIRA who survived into adulthood without IL-1-blocking treatment, suggesting that, if untreated, the mortality may be higher than estimated based on patients who have so far been diagnosed. DIRA can be and has historically been misdiagnosed as infectious osteomyelitis with pustulosis and systemic inflammation; thus, treatment with antibiotics is often initiated. However, therapeutic intervention with corticosteroids or, more recently, since the discovery of the genetic cause, IL-1-blocking treatment with anakinra can be life saving. IL-1 blockade leads to rapid and durable clinical and inflammatory remission in DIRA patients.

The short-acting IL-1 inhibitor anakinra, which is a "recombinant" IL-1Ra, the molecular replacement of the missing endogenous protein, needs to be subcutaneously injected daily $(10,11)$. The injections are painful, and drug reactions to anakinra have been seen in 2 DIRA patients (12). The absence of endogenous protein in most patients raises concerns about increased antigenicity of the recombinant protein. Rilonacept is a long-acting soluble decoy receptor, engineered as a dimeric fusion protein that consists of the ligand-binding domains of the extracellular portions of the human IL-1 receptor linked to the Fc region of human IgG1 that binds to both IL-1 ILs, IL-1 $\alpha$ and IL-1 $\beta$, and prevents their interaction with the IL-1 cell surface receptor (13-15). Its half-life is approximately 7.5 to 8 days, and it is administered by a weekly injection. The FDA approved rilonacept in 2008 for the treatment of adults and children 12 years of age and older with cryopyrin-associated periodic syndromes (CAPS), including familial cold autoinflammatory syndrome and Muckle-Wells syndrome. The drug safety is well tolerated. No serious adverse events have been reported with its use in the literature $(16,17)$. Given that DIRA requires life-long treatment, the potential benefit of weekly injections over current standard of care with daily injections, may be substantial. For these reasons, the current study was designed to evaluate the use of rilonacept in patients with DIRA.

\section{Results}

\section{Historical data for pretreatment and anakinra treatment}

Table 1 summarizes the demographic characteristics and clinical presentations of the DIRA patients before treatment with anakinra was started (see Figure 1, for study schedule). All patients presented at birth to 2 weeks of life with fetal distress, pustular rashes, osteomyelitis, and systemic inflammation (elevated acute-phase reactants and leukocytosis). Onychomadesis (shedding of finger nails) was described in 4 of 6 patients. Inflammatory bone lesions in the period before anakinra treatment included osteomyelitis of ribs, with rib widening on the x-rays $(n=4)$. All 6 patients had periostitis affecting several long bones, and multifocal osteolytic lesions (Supplemental Figure 1; supplemental material available online with this article; https://doi.org/10.1172/jci.insight.94838DS1). CNS vasculitis/vasculopathy occurred in one patient at the age of 2 months. Three patients developed thrombosis at the site of catheter insertion. In the period before anakinra treatment, which spanned from 2 months to 18 months, all 6 patients had between 3 and 5 prolonged hospitalizations, with an average length of about 107 days in total hospitalization. All of these patients were treated with antibiotics with no response. Intravenous immunoglobulin and corticosteroids partially controlled the symptoms (Table 1). All patients accrued marked bone damage before anakinra was started. Sequelae of sterile osteomyelitis were as follows. Two patients had odontoid destruction caused by osteomyelitis of the odontoid that led to atlantoaxial joint instability requiring cervical spine surgeries (patient 3 and patient 5). Two patients developed kyphotic deformities (gibbus formation) from vertebral 
Table 1. Baseline demographic and clinical characteristics

\section{Characteristic}

Age of disease onset (d)

Age group (no. [\%]) $)^{A}$

Value

$15.3 \pm 22.60$

$4.8 \pm 1.5$

0

$\operatorname{Sex}($ no. [\%])

$0-2 \mathrm{yr}$
$2-4 \mathrm{yr}$
$4-6 \mathrm{yr}$

$3(50)$

Race or ethnic group (no. [\%])

$$
\text { Male (no. [\%]) }
$$

White Hispanic

Laboratory abnormalities (minimum - maximum) ${ }^{\mathrm{B}}$

$$
\begin{aligned}
& \text { WBC }(\mathrm{k} / \mu \mathrm{l}) \\
& \text { Platelets }(\mathrm{k} / \mu \mathrm{l}) \\
& \text { ESR }(\mathrm{mm} / \mathrm{hr}) \\
& \operatorname{CRP}(\mathrm{mg} / \mathrm{l})
\end{aligned}
$$

14.7-37.9

574-986

47-113

$17.5-192.5$

Treatments

$$
\begin{gathered}
\text { Oral corticosteroids }{ }^{\mathrm{c}} \text { (no. [\%]) } \\
\text { Mean dose of corticosteroids pre-rx (mg/kg/d) }{ }^{\mathrm{D}} \\
\text { DMARDs prior to anakinra } \\
\text { Anakinra (no. [\%]) } \\
\text { Anakinra treatment duration (mo.) } \\
\text { Anakinra dose (mg/kg/d) } \\
\text { Antibiotics (no. [\%]) } \\
\text { Antivirals (no. [\%]) } \\
\text { Antifungals (no. [\%]) } \\
\text { Systemic inflammation }{ }^{\mathrm{F}} \\
\text { Anemia }{ }^{\mathrm{C}}
\end{gathered}
$$

38-65 mo.

$3-4.5$

$6(100)$

2 (34)

\section{Hospitalizations prior to anakinra}

$$
\begin{aligned}
& \text { Mean number per patient } \\
& \text { Length of stay (in d) }
\end{aligned}
$$

\section{$3.3 \pm 1.21$ \\ $107 \pm 75$}

Skin involvement

$$
\text { Pustulosis }
$$

Onychomadesis 4(66)

Vasculitis

$\begin{array}{ll}\text { CNS } & 1(16) \\ \text { Skin }^{H} & 1(16) \\ \text { vein thrombosis } & 3(50)\end{array}$

Osteomyelitis

$$
\text { Deep vein thrombosis }
$$

3 (50)

\begin{tabular}{cc}
\hline Os odontoid osteomyelitis & $2(34)$ \\
Vertebral osteomyelitis & $2(34)$ \\
Ribs & $5(84)$ \\
Hip & $4(66)$ \\
Long bones & $3(50)$ \\
Short bones (phalanx) & $2(34)$ \\
Periosteitis & $6(100)$
\end{tabular}

Bone damage

Vertebral block formation

$\begin{array}{cc}\text { Cervical spine } & 2(34) \\ \text { Thoracic spine } & 2(34) \\ \text { Cervical spine stabilization surgeries } & 2(34)\end{array}$

${ }^{A}$ Age at the time of enrollment into the rilonacept study. ${ }^{B}$ Laboratory abnormalities before treatment with anakinra. ${ }^{\mathrm{C} P r e d n i s o n e ~ o r ~ e q u i v a l e n t . ~}{ }^{\mathrm{P} P r e t r e a t m e n t}$

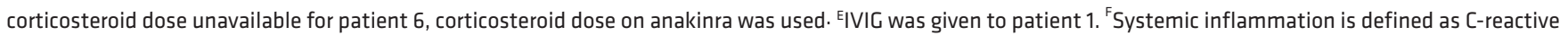
protein (CRP) $>3.0 \mathrm{mg} / \mathrm{l}$ and/or erythrocyte sedimentation rate (ESR) $>25 \mathrm{~mm} / \mathrm{hr}$. ${ }^{\mathrm{C}}$ Anemia ( $\mathrm{Hb}<9.7 \mathrm{~g} / \mathrm{dl}$ ). ${ }^{\mathrm{H}}$ Patient 1 was noted to develop pathergy like skin lesions in response to needle injections. The same patient had a bone biopsy on an osteolytic lesion; blood vessel close to the bone was read as neutrophilic vasculitis. WBC, white blood cell count; DMARDs, disease-modifying antirheumatic drugs. Numbers in parentheses indicate percentage calculated from a total of 6 patients. 


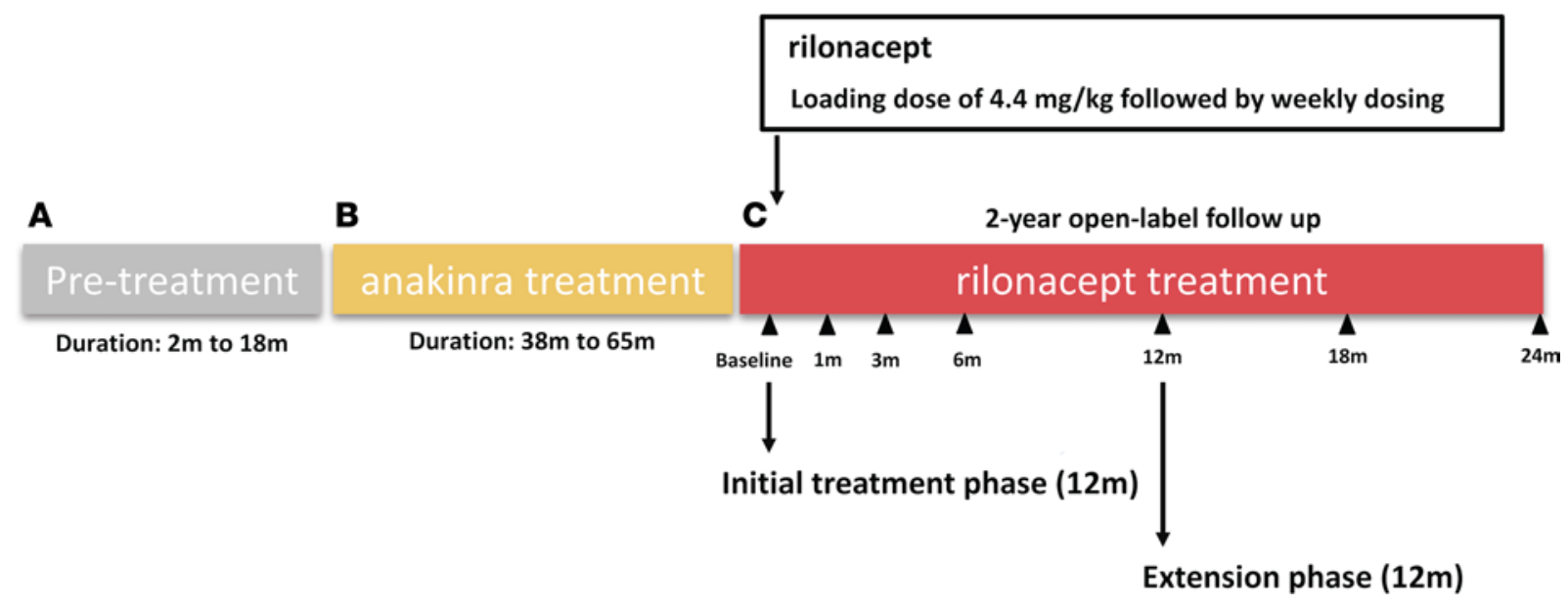

Figure 1. Study design. (A) Length of time in months (minimum to maximum) patients were untreated. During this time they acquired the organ damage listed in Table 1. (B) Length of time in months patients were treated with the recombinant IL-1 receptor anakinra and prior to enrollment into the open-label study. (C) Open-label study overview. Anakinra was discontinued prior to initiation of the study. Loading dose of rilonacept was given at $4.4 \mathrm{mg} / \mathrm{kg} /$ wk, followed by a maintenance dose of $2.2 \mathrm{mg} / \mathrm{kg} / \mathrm{wk}$. Patients were seen every 1 to 3 months for the first 6 months of the initial phase and then every 6 months for the duration of the study. The length of the open-label study was 2 years.

collapse secondary to vertebral osteomyelitis (patient 3 and patient 6). One patient had developed leg length discrepancy (patient 1).

Anakinra was started in all patients between the ages of 2 months and 18 months, mean age $9.1 \pm 6.24$ months, and patients were on treatment between 38 and 65 months (mean $47.08 \pm 9.94$ months) prior to enrolling into this study. On anakinra treatment, the acute-phase reactants (erythrocyte sedimentation rate [ESR] and C-reactive protein [CRP]) and the white blood cell count, hemoglobin, and platelet count normalized in all patients (Supplemental Table 1 and Supplemental Figure 2).

\section{Rilonacept treatment resulted in continued inflammatory remission in DIRA patients}

Between 2013 and 2015, 6 DIRA patients with a mean age of 4.8 years (range 3.3 to 6.2 years) were enrolled to receive rilonacept. At enrollment, all patients were on a mean dose of anakinra of $3.58 \pm 0.58 \mathrm{mg} / \mathrm{kg} / \mathrm{d}$ (minimum $3 \mathrm{mg} / \mathrm{kg} / \mathrm{d}$, maximum $4.5 \mathrm{mg} / \mathrm{kg} / \mathrm{d}$ ). After a 24 -hour washout period, all patients received an initial bolus of $4.4 \mathrm{mg} / \mathrm{kg} /$ dose of rilonacept and were discharged on $2.2 \mathrm{mg} / \mathrm{kg} / \mathrm{wk}$. All except one patient (patient 6) required a rilonacept dose escalation to $4.4 \mathrm{mg} / \mathrm{kg} / \mathrm{wk}$ due to minor flares presenting with micropustules in areas of hyperkeratosis (Supplemental Figure 3). The development of micropustules resulted in a temporary increase in the diary scores at the 3 months visit (Figure 2). When the rilonacept dose was adjusted to $4.4 \mathrm{mg} / \mathrm{kg} / \mathrm{wk}$, the micropustules improved and all patients stayed in inflammatory remission throughout the study period of 2 years (Figure 2). One patient (patient 2) continued with sporadic presence of 1 to 5 micropustular lesions throughout the duration of the study. She was not considered to fulfill flare criteria, as the skin lesions were intermittent, nonpruritic, and otherwise asymptomatic and did clinically not warrant any further dose increase. She continued on rilonacept at $4.4 \mathrm{mg} / \mathrm{kg} / \mathrm{wk}$.

Rilonacept at a dose of $2.2 \mathrm{mg} / \mathrm{kg}(n=1)$ and $4.4 \mathrm{mg} / \mathrm{kg}(n=5)$ per week provided sustained control of clinical symptoms and inflammatory markers. Nonclinically significant temporary increases in inflammatory markers, including CRP and ESR, were seen at the 3-month, 12-month, and 24-month visits (Figure 2). At 3 months, one patient (patient 2) developed a transient disease flare, with micropustules on the left elbow, left forearm, left knee, and bilateral lower legs, with an increase in acute-phase reactants (ESR of $25 \mathrm{~mm} / \mathrm{hr}$ and CRP of $7.07 \mathrm{mg} / \mathrm{l}$ ). The flare occurred in the setting of an otitis media and a fixed drug reaction to acetaminophen. The disease flare led to a permanent increase in rilonacept to $4.4 \mathrm{mg} / \mathrm{kg} / \mathrm{wk}$ for the entire study period. Patient 4 had 2 infections, one at the 12-month visit and one at the 24-month visit. At 12 months, she presented with an episode of otitis media that was treated with antibiotics and was associated with a CRP of $8.9 \mathrm{mg} / 1$, and, at 24 months, she presented with an upper respiratory infection associated with a CRP of $3.1 \mathrm{mg} / 1$. The rilonacept dose remained unchanged at $4.4 \mathrm{mg} / \mathrm{kg} / \mathrm{wk}$ at both times. After the initial dose increases that all occurred within 3 months of starting rilonacept, diary 
A
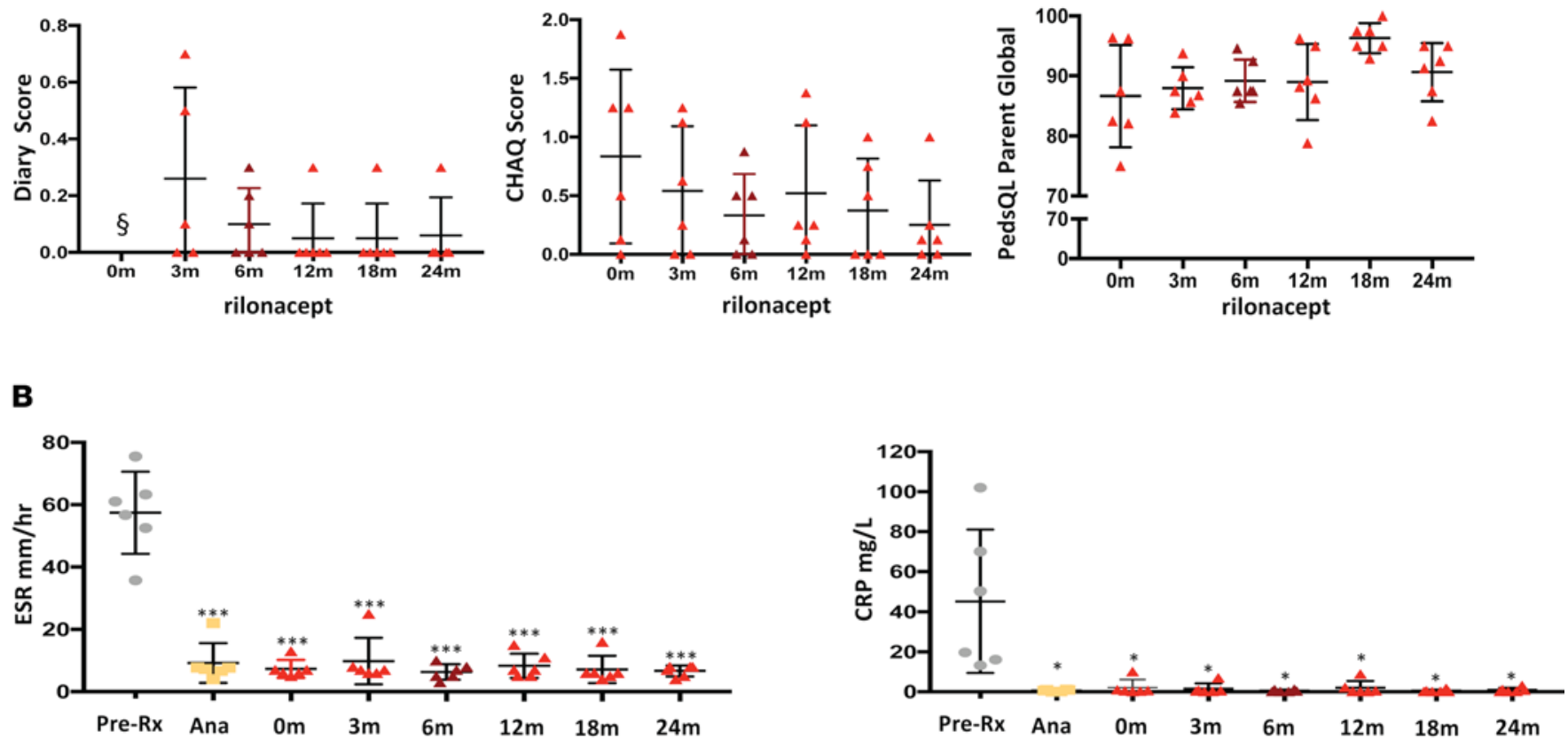

C
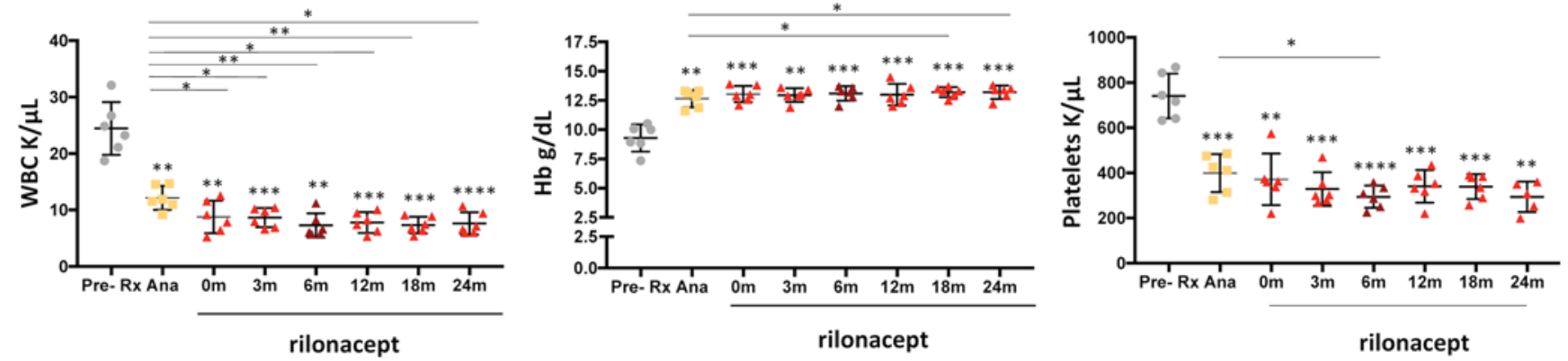

Figure 2. Clinical and laboratory responses in patients with deficiency of IL-1 receptor antagonist treated with rilonacept. (A) Diary scores, Childhood Health Assessment Questionnaires (CHAQ) scores, and Pediatric Quality of Life (PedsQL) assessments were obtained at the baseline visit and after 3, $6,12,18$, and 24 months on rilonacept $(n=6)$. $\S$ denotes that the diary score was 0 at baseline. The diary, CHAQ, and PedsQL scores did not significantly change from baseline. The transient elevation on the diary score at 3 months reflects the presence of micropustules in keratinized skin areas (i.e., elbow and knees). (B) C-reactive protein (CRP) level and erythrocyte sedimentation rate (ESR) were compared between the pretreatment period, the anakinra treatment period, and the visits after initiation of rilonacept (baseline visit and after 3, 6, 12, 18, and 24 months). (C) White blood cells count (WBC), hemoglobin $(\mathrm{Hb})$, and platelet count changes pretreatment, on anakinra, and on the respective rilonacept visits (baseline and after 3, 6, 12, 18, and 24 months) were compared. Comparisons among pretreatment, on anakinra, and respective rilonacept visits were made using paired $t$ tests $\left({ }^{*} P<0.05\right.$, ${ }^{* *} P<0.01$, $\left.{ }^{* * *} P<0.001,{ }^{* * *} P<0.0001\right)$. Dots represent individual patients, and lines show the mean \pm SD. Asterisks over values at test points indicate comparisons between pretreatment and anakinra as well as pretreatment and rilonacept. Asterisks over lines indicate comparisons between anakinra versus rilonacept.

scores remained low and inflammatory markers remained normal (except during periods of infections). All patients in the rilonacept study remained in remission, and none of the patients fulfilled flare criteria after rilonacept dose adjustments were made (see Methods for definition of flare). The Childhood Health Assessment Questionnaire (CHAQ) showed improvement, from a mean of $0.83 \pm 0.74$ at baseline (on anakinra) to $0.25 \pm 0.37$ at 24 months $(P=0.06)$. All patients, except patient 3 and patient 4 , had clinically significant improvement in their CHAQ scores. Patient 3 continued to have a disability index of 0.125 as a result of bone damage from vertebral fusion in thoracic spine, resulting in kyphosis, and atlantoaxial instability that required surgical repair. The Pediatric Quality of Life Inventory (PedsQL) score showed improvement from $86.6 \pm 8.51$ to $90.6 \pm 4.85(P=0.07)$; the improvement in PedsQL score reached statistical significance at the 18 -month visit $(P=0.05)$. At the end of the rilonacept study, the PedsQL score dropped, which might be explained by the parents'/patient's apprehension to restart daily anakinra 
Table 2. Height and weight $Z$ score change on treatment

\begin{tabular}{|c|c|c|c|c|c|}
\hline & $\begin{array}{l}\text { Pretreatment (mean } \pm \\
\text { SD) }\end{array}$ & Anakinra (mean \pm SD) ${ }^{\mathrm{B}}$ & Rilonacept (mean \pm SD) ${ }^{c}$ & $P$ value $^{\mathrm{D}}$ & P value ${ }^{E}$ \\
\hline Height & $-2.07 \pm 1.13$ & $-1.10 \pm 0.41$ & $-1.22 \pm 0.48$ & 0.04 & 0.04 \\
\hline Weight & $-3.17 \pm 2.07$ & $-0.59 \pm 0.62$ & $-0.48 \pm 0.64$ & 0.04 & 0.03 \\
\hline \multicolumn{6}{|c|}{$\begin{array}{l}{ }^{\mathrm{A}} \text { Historical values collected from a period before patients were started on anakinra. }{ }^{\mathrm{B}} \text { Measurements on anakinra treatment represent values that were } \\
\text { collected at the time of enrollment and prior to rilonacept treatment. }{ }^{C} \text { Height and weight } Z \text { scores obtained on rilonacept reflect values collected at the } \\
\text { 24-month visit. }{ }^{D} P \text { value comparing pretreatment } Z \text { scores to anakinra treatment } Z \text { scores. }{ }^{E} P \text { value comparing pretreatment } Z \text { scores to rilonacept } Z \text { scores. }\end{array}$} \\
\hline
\end{tabular}

injections; interestingly, the one patient (patient 5) who received insurance approval to continue rilonacept reported stable improvement in PedsQL score from 92.9 to 95.

\section{Linear growth and bone mineral density normalize with anti-IL-1 treatment and remain normal on rilonacept treatment}

Linear growth and weight. Five patients were born with normal height and weight; one patient, who was a product of a twin pregnancy at 37 weeks gestation, had a low birth weight of $1.98 \mathrm{~kg}$. Within weeks of disease onset, all 6 patients had weight decreases to below the third percentile. Five patients' heights dropped to below the third percentile and one patient to below the fifth percentile by the time they started anakinra. Mean height and weight $Z$ scores increased while patients were on anakinra and further increased on rilonacept (comparing pretreatment with treatment with rilonacept for height and weight, $P<0.05$, respectively) (Table 2 and Figure 3).

Bone mineral density. Bone mineral density also improved on IL-1-blocking treatment (Figure 4). Two patients (patient 1 and patient 4), who were age 9 months and 18 months at time of anakinra initiation, had dual-energy x-ray absorptiometry (DEXA) scans within 4 months and 2 days of anakinra treatment, respectively. Both were osteoporotic with anteroposterior spine $Z$ scores of -4.2 and -4.5 , respectively. Two patients (patient 2 and patient 3), who were age 4 months and 15 months at the time of anakinra initiation, had DEXA scans at 8 and 15 months on anakinra treatment. Both had osteopenia with anteroposterior spine $Z$ scores of -2.1 and -2.2 , respectively. Two patients (patient 5 and patient 6), who were 2 months and 9 months at the time of anakinra treatment, had DEXA scans after 3 years and 2 months and 3 years and 3 months on anakinra. They had normal $Z$ scores of -1.1 and -1.0 , respectively. These data illustrate the rapid loss of bone mineral density with active untreated DIRA and the ability to improve and normalize bone mineral density on IL-1 blockade with anakinra treatment. At the time of initiation of rilonacept treatment, all patients had normal bone mineral densities, with $Z$ scores of $-0.08 \pm 1.23$ (minimum -1.1 , maximum 2.0), and after 1 year on rilonacept treatment, the mean $Z$ scores remained normal at $-0.12 \pm 1.26$ (minimum -1.5 , maximum 2.0), suggesting further stabilization of normal growth and bone mineral density on rilonacept.

\section{Rilonacept pharmacokinetic parameters and anti-drug antibody development}

The patients on study were between 2 and 7 years of age; pharmacokinetic and safety data had not previously been assessed in this age group. Supplemental Figure 4 shows the individual functional rilonacept concentration-time profiles for the six patients enrolled in the study. Supplemental Figure 5 shows the mean $( \pm S D)$ concentration-time profiles by dose level. Between months 6 and 24, mean steady-state trough concentrations were $63.5-74.0 \mathrm{mg} / 1$ for the $4.4 \mathrm{mg} / \mathrm{kg}$ dose. For the one patient who remained on the $2.2 \mathrm{mg} / \mathrm{kg}$ dose, steady-state trough concentrations ranged between 22.2 and $36.5 \mathrm{mg} / 1$. These data are consistent with linear pharmacokinetics, as the steady-state trough concentrations are approximately dose proportional. Mean steady-state clearance for the $4.4 \mathrm{mg} / \mathrm{kg} / \mathrm{wk}$ dose was estimated at the 24-month time point to be $0.00991 / \mathrm{kg} / \mathrm{d}$ from the ratio of dose/concentration. Although pretreatment anti-drug antibodies (ADAs) were measured in 3 patients at baseline (patients 2, 3, and 6), most likely due to higher assay background in these samples, no rilonacept treatment-emergent ADAs occurred in any patient and all predose ADAs titers turned negative on rilonacept treatment. 

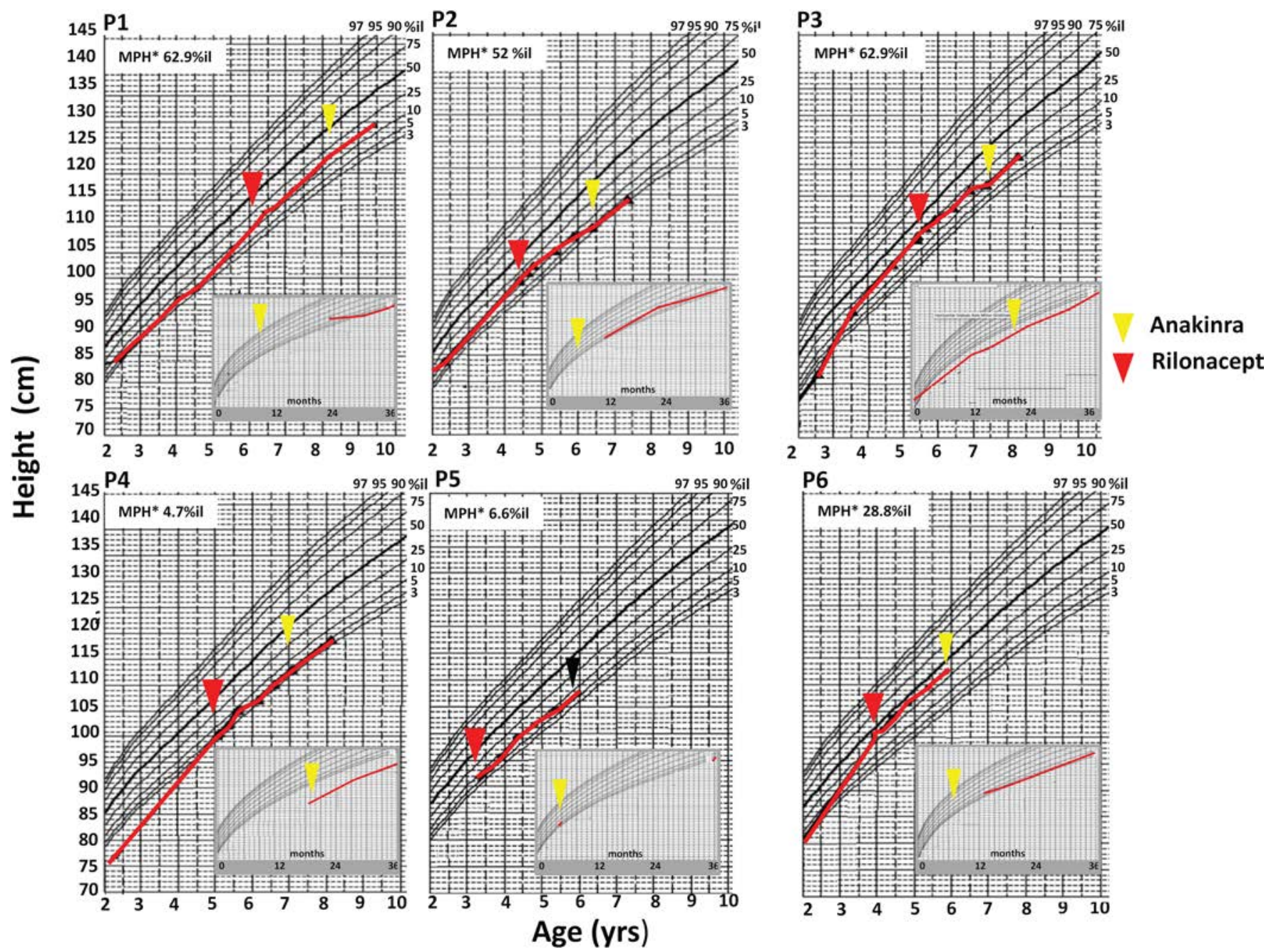

Figure 3. Linear growth on IL-1-blocking treatment is shown for each patient enrolled. Historical data from prior to the initiation of rilonacept (red arrowhead indicates start of rilonacept) were collected and graphed. The inset in each graph shows height obtained between 0 and 36 months of age. The yellow arrowhead in the inset denotes initiation of anakinra treatment; in the big graph, the yellow arrowhead indicates reinitiation of anakinra treatment after completion of the rilonacept study. The graph shows heights collected from the ages of 2 years until the end of rilonacept treatment ( 2 years of treatment). Patient 5 remains on rilonacept after the trial (the end of rilonacept study indicated by the black arrowhead). Midparental height (percentiles) are indicated in the top left corner of each graph. P1, patient 1; P2, patient 2; P3, patient 3; P4, patient 4; P5, patient 5; P6, patient 6.

\section{Safety assessments on rilonacept treatment}

Rilonacept was well tolerated, no serious adverse events were reported, and none of the patients met protocol adverse event criteria for rilonacept dose reductions or permanent discontinuation. There appeared to be no clinically significant increase in number or severity of adverse events at dosages of $4.4 \mathrm{mg} / \mathrm{kg} / \mathrm{wk}$ as compared with the $2.2 \mathrm{mg} / \mathrm{kg} / \mathrm{wk}$ dose, but only one patient stayed on the $2.2 \mathrm{mg} / \mathrm{kg} / \mathrm{wk}$ dose for the duration of the study. All subjects had at least one treatment-emergent adverse event (TEAE). The most common events were upper respiratory infection (6 of 6), otitis media, and rash (4 of 6), followed by pharyngitis, rhinorrhea, gastrointestinal symptoms, and an allergic reaction to food and acetaminophen (Table 3 and Supplemental Table 2). All TEAE were mild or moderate in severity (Table 3).

\section{Discussion}

DIRA is an ultra-rare autoinflammatory disease characterized by systemic inflammation, pustulosis, and sterile osteomyelitis $(2,3)$. It is caused by genetic mutations that result in the loss of functional IL-1Ra. Rapid response to treatment with recombinant IL-1Ra, anakinra, clinically validates the key role of IL-1 in causing the severe clinical phenotype of DIRA. This study showed that rilonacept treatment enabled sustained ( 2 year) clinical and inflammatory remission in 6 DIRA patients who had been previously treated 


\section{Table 3. Treatment-emergent adverse events by severity}

\begin{tabular}{|c|c|c|}
\hline Adverse event ${ }^{A}$ & Mild $^{B}(n=6)$ & Moderate $(n=6)$ \\
\hline Number of TEAEs & 61 & 23 \\
\hline \multicolumn{3}{|l|}{ Infectious TEAEs } \\
\hline $\begin{array}{l}\text { Upper respiratory } \\
\text { infection }\end{array}$ & 6 & 2 \\
\hline Otitis media & 3 & 3 \\
\hline Otitis externa & 1 & 0 \\
\hline Pharyngitis & 0 & 3 \\
\hline Rhinorrhea & 3 & 0 \\
\hline Sinusitis & 0 & 1 \\
\hline Pneumonia & 1 & 0 \\
\hline Viral illness & 1 & 0 \\
\hline Rash $^{\mathrm{C}}$ & 4 & 2 \\
\hline \multicolumn{3}{|l|}{ Noninfectious TEAEs } \\
\hline Abdominal pain & 0 & 2 \\
\hline Gastroenteritis & 2 & 0 \\
\hline Vomiting & 2 & 0 \\
\hline Allergic reaction ${ }^{\mathrm{D}}$ & 1 & 1 \\
\hline Fever & 1 & 1 \\
\hline Headache & 2 & 0 \\
\hline CPK increased & 2 & 0 \\
\hline Arthralgia & 2 & 0 \\
\hline Thrombocytosis & 1 & 0 \\
\hline
\end{tabular}

${ }^{A}$ All suspected and nonsuspected TEAEs are included. A subject with multiple TEAEs is counted once for the same event term, per severity, i.e., a subject that had more than one mild and/or moderate upper respiratory infection during the study is counted once in the mild column and once in the moderate column. ${ }^{\mathrm{B}}$ The following noninfectious adverse events occurred in one patient only and were rated as mild: dry skin, hypercholesterolemia, constipation, muscle soreness, photosensitivity, pruritus, seborrheic dermatitis, eczema, and allergic conjunctivitis. One patient had an injection site reaction upon reinitiation of anakinra after discontinuation of rilonacept that was reported on the study. 'Rashes include those associated with infections and the micropustular lesions that responded to rilonacept dose increase. ${ }^{D}$ Food allergy ( $n$ $=1$ ) and fixed drug reaction to acetaminophen $(n=1)$. TEAEs, treatment-emergent adverse events coded by MedDRA (TEAEs are classified as mild to moderate according to CTCAE-v.04 criteria); $n$ = number of patients. with anakinra. Rilonacept treatment was generally safe, and the weekly injections were well tolerated.

Treatment of DIRA is lifelong, as the removal of IL-1-suppressive therapy leads to escalating disease flares that can be triggered by infections and stress (2) (our unpublished observations). Several historical cases of untreated patients illustrate the need to rapidly recognize DIRA and to initiate antiIL-1-blocking therapy. If untreated, rapid escalation to a SIRS can lead to shock and organ failure (18); SIRS is a rapidly progressing hyperinflammatory state that is also seen in patients with other untreated autoinflammatory diseases. Before IL-1-blocking treatment was available, DIRA patients had prolonged hospitalizations and severe illness. Anakinra (recombinant IL-1Ra) has been lifesaving; however, anakinra is administered by daily subcutaneous injections, which can be painful and cumbersome. Allergic reactions to anakinra have been reported in 2 DIRA patients (12). Rilonacept can thus be an alternative for patients who develop allergic reactions to anakinra.

Rilonacept is a fusion protein derived from human sequence (IL-1R, ACP, IgG FC). Rilonacept is injected weekly and has been approved by the FDA for the treatment of patients with the milder forms of the rare autoinflammatory diseases syndrome CAPS in the US (https://www.regeneron. com/arcalyst-injection), namely, familial cold-induced autoinflammatory syndrome and Muckle Wells syndrome, who are older than 12 years of age $(16,17,19)$. Dosing and efficacy have not been assessed in neonatal-onset multisystem inflammatory disease (NOMID), a severe and sporadic form of CAPS. Rilonacept has also been used in the treatment of patients with systemic-onset juvenile idiopathic arthritis (JIA) (20), and a multicenter international phase III trial in 1,315 patients with gout showed that rilonacept significantly reduces the risk of gout flares in patients receiving uric acid-lowering therapy (21-23). We hypothesized that capture and functional inactivation of circulating IL- $1 \alpha$ and IL-1 $\beta$ by rilonacept would be functionally equivalent to the absent or nonfunctional IL-1Ra in DIRA patients, thus substituting for the absent function of IL-1Ra by binding to IL- $1 \alpha$ and IL-1 $\beta$. DIRA and CAPS both are characterized by dysregulation and overactivity of the IL-1 cytokine system. The spectrum of symptoms in DIRA is different from that of CAPS and the presence of lesions in hyperkeratotic areas suggested a potential role for IL- $1 \alpha$ in the disease pathogenesis of DIRA, while the spectrum of symptoms in NOMID and CAPS would be predominantly IL- $1 \beta$ mediated. In contrast to IL- $1 \beta$, the pathogenic role of IL- $1 \alpha$ is not well understood. Many organ-specific cells express IL- $1 \alpha$, thus raising the question of its potential role in tissue homeostasis (24). Since models for in vitro "mimics" of relevant tissue inflammation (skin and bone in DIRA patients) and damage do not currently exist, and Il1rn-knockout mice do not develop the relevant tissue inflammation seen in DIRA patients, it is currently impossible to reliably determine the tissue effect of unopposed IL-1 $\alpha$. Data on organ inflammation and tissue expression of IL-1 $\alpha$ provide the basis for concern regarding potential unopposed actions of IL-1 $\alpha$ that may contribute to tissue inflammation, including the development of destructive osteomyelitis and the neutrophilic pustulosis that are seen in all DIRA patients but not in any of the Illrn-knockout murine models, further suggesting that tissue expression or regulation of IL- $1 \alpha$ and IL-1 $\beta$ may differ in mice and humans. To block all potential inflammatory sources that may lead to tissue damage, we chose rilonacept for its ability to mimic the actions of anakinra most closely. We hypothesized that IL-1 $\alpha$ could play a role in the tissue manifestations leading to osteoclast activation and contribute to osteomyelitis and bone damage, osteopenia, and osteoporosis, thus regulating bone homeostasis (25), and to the development of skin pustulosis, as IL-1 $\alpha$ and IL-1Ra are highly expressed in keratinocytes (26), and IL-1Ra is absent in patients with DIRA. Autocrine secretion of IL-1 $\alpha$ can lead to impaired migration and differentiation (27) and altered 


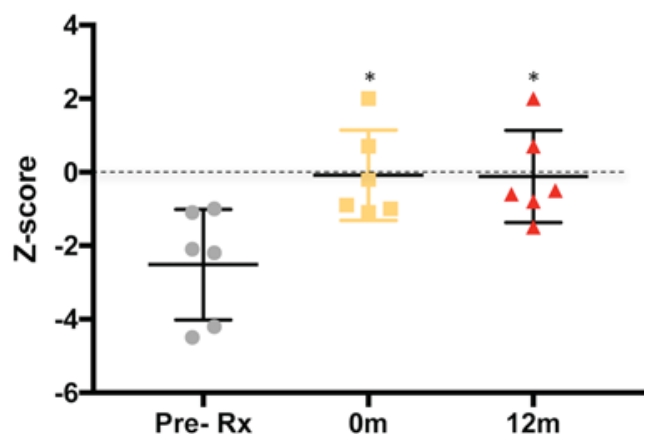

Figure 4. Dual-energy X-ray absorptiometry. Mean $Z$ scores of anteroposterior lumbar spine were used for comparisons. Prior to treatment with anakinra, two patients had osteoporosis, and another two had osteopenia documented within the first year of anakinra initiation. $Z$ scores normalized after treatment with anakinra from $-2.52 \pm 1.51$ to $-0.08 \pm 1.23(P<0.05)$ $Z$ scores continued to be normal after 12 months of treatment with rilonacept (mean $Z$ score $-0.12 \pm 1.26$ ) $(P<0.05$ versus pretreatment period). Comparisons between pretreatment, on anakinra, and respective rilonacept visits were made using paired $t$ tests $\left({ }^{*} P\right.$ $<0.05)$. Dots represent individual patients, and lines show the mean $\pm \mathrm{SD}$. antimicrobial responses (28). Obviously, more data are needed to determine the long-term effect of presumed unopposed IL-1 $\alpha$ signaling on the development of subclinical organ inflammation and potential organ damage. Although, recently, a DIRA patient was started on canakinumab, an IL-1 $\beta$-antagonizing antibody with reported short-term benefit (6), there are no long-term outcome data currently, that suggest that IL-1 and IL-1 $\beta$ control is better than IL-1 $\beta$ control alone; however, in all patients on chronic IL-1 inhibition, bone mineral density and skin lesions should be closely monitored.

Rilonacept is FDA approved for the treatment of CAPS in children older than 12 years of age. Efficacy, dosing, and safety had not been established for CAPS patients less than 12 years of age. Our study is the first to our knowledge to evaluate the safety, efficacy, and pharmacokinetics of rilonacept in DIRA patients less than 5 years of age. The pharmacokinetic profiles were consistent with expectations based on data obtained in CAPS patients older than 12 years of age as well as systemic JIA (sJIA) patients aged 1-18 years. The clearance of rilonacept in children under 5 years was previously estimated in sJIA patients to be $0.012(0.009-0.054) 1 / \mathrm{kg} / \mathrm{d}$ (29); the data obtained in children with DIRA are consistent with this estimate. Albumin has been shown to be a significant covariate on rilonacept clearance, but marked increases in clearance were only observed at albumin concentrations below $4 \mathrm{~g} / \mathrm{dl}$ (29). Available data from patients in the current study show that albumin ranged between 4.0 and $4.9 \mathrm{~g} / \mathrm{dl}$. Rilonacept was well tolerated. No serious adverse events occurred. All TEAE were mild or moderate in severity. No patient discontinued the study due to TEAE. As seen in other autoinflammatory diseases, infections, including upper respiratory infections and otitis media, can induce flares of the underlying disease, with clinical symptoms of DIRA, including pustulosis, fever, and elevation of inflammatory markers, which are not seen in "healthy" children without DIRA in the context of a viral infection. However, these disease flares have been mild and transient and respond to transient increase in the IL-1-blocking agents. The fact that infection-associated disease flares are milder and more manageable on IL-1-blocking treatments indicates a potential regulatory role for IL-1 blockade in reducing priming of hematopoietic and possibly even organ-specific cells.

Although the clinical responses to rilonacept were comparable to those seen with anakinra, there was a clear parent and patient preference for staying on a weekly injection versus daily injections. Interestingly, the apprehension of returning to daily injections was reflected in the PedsQL score at the last visit, when 5 of 6 patients who were transitioned back to anakinra reported lower PedsQL scores. The normalization of linear growth with children showing catch-up growth and the stabilization in bone mineral density with normalization of $Z$ scores, suggests that treatment with rilonacept propagates normal growth and maintains normal bone mineral density development. Whether rilonacept would be effective in the treatment of other forms of sterile osteomyelitis, including Majeed syndrome $(30,31)$, and genetically undefined or polygenic (32) forms of osteomyelitis remains to be determined.

In summary, our study shows that rilonacept is well tolerated and effective in maintaining control of disease manifestations of DIRA over a 2-year period. Favorable effects on clinical well being, normalization of inflammatory markers, maintenance of bone mineral density, and improvement in growth rates, and the rapid development of flares in the absence of adequate treatment (18), suggest that it is reasonable to expect that rilonacept treatment could initiate effective disease control in treatment-naive patients.

\section{Methods}

See the Supplemental Methods for additional information.

\section{Patients}

Genetically confirmed DIRA patients on treatment with anakinra were eligible to be enrolled into the study (ClinicalTrials.gov NCT01801449). The first 5 patients had to be older than 2 years before younger ( $>3$ months) treatment-naive patients could be enrolled, as safety data had only been gathered for patients $\geq 5$ years of age. All patients had active disease prior to initiation of anakinra treatment. 
Their case histories have been published $(3,4,18,33,34)$. None of the patients enrolled were on disease-modifying antirheumatic drugs, including methotrexate or oral steroids, at the time of enrollment. Patients had to be on stable anakinra doses during the 2-week period prior to the screening visit. Anakinra was discontinued 24 hours before initiation of rilonacept treatment.

\section{Study design and treatment}

This study was initially designed as a 1-year study and was subsequently extended to 2 years. The rilonacept study had five main phases: (a) screening (subjects who received anakinra); (b) initial dosing of rilonacept (following a 24-hour washout of anakinra); (c) initial assessment for dose escalation requirement (0-3 months); (d) treatment phase (3-12 months); and (e) extension phase (12-24 months). The study was conducted between 2013 and 2015 (Figure 1). Prior to the screening visit, daily diary data were collected during a 2-week period (baseline). Concomitant antiinflammatory drug and corticosteroid doses had to be stable for 2 weeks. After enrollment, anakinra was stopped for 24 hours before rilonacept initiation. All patients received a loading dose of rilonacept of $4.4 \mathrm{mg} / \mathrm{kg}$ subcutaneously, followed by once weekly subcutaneous injections of $2.2 \mathrm{mg} / \mathrm{kg}$ rilonacept (up to a maximum of $160 \mathrm{mg} / \mathrm{wk}$ ). Patients were evaluated at 1-, 3-, 6-, and 12-month visits at the NIH. Follow-up telephone calls were scheduled 2 weeks after administration of the initial rilonacept dose and after 9 months. If applicable (i.e., after a dose increase), subjects were evaluated by their local physicians in their respective home countries, including Brazil, Canada, and USA. Safety laboratory measurements were drawn locally, and the results were transmitted to the NIH. However, subjects requiring dose escalation due to clinical flares were required to be evaluated at the NIH. In the extension period, outcomes were assessed at 15 (telephone call), 18, and 24 months, and safety was monitored throughout.

Inflammatory remission. Inflammatory remission criteria were defined as all of the following: a diary score of $<0.5$ (reflecting no fever, skin rash, or bone pain), normal acute-phase reactants ( $\mathrm{CRP}<0.5 \mathrm{mg} /$ dl), and no objective skin rash or radiological evidence of active bone lesions on $\mathrm{x}$-ray.

Flare criteria. Flare criteria were defined as any of the following: fever (oral temperatures $>38^{\circ} \mathrm{C}$ on 3 or more occasions in 1 week that were not infection related), a DIRA-associated skin rash (i.e., localized or generalized pustular skin lesions that can present in areas of mechanical stress), clinical and or radiographic signs and symptoms of bone disease (including presence of periostitis and or osteolytic lesions on $\mathrm{x}$-ray or MRI), increase in acute-phase reactants (CRP $>0.5 \mathrm{mg} / \mathrm{dl}$ not associated with an infection); or any other potential DIRA-associated acute inflammatory events, such as CNS vasculitis or inflammatory pulmonary lesions.

\section{Potential dose escalation}

Subjects who remained in inflammatory remission continued rilonacept injections of $2.2 \mathrm{mg} / \mathrm{kg} / \mathrm{wk}$. Subjects not meeting the inflammatory remission criteria or meeting flare criteria could be dose escalated to 4.4 $\mathrm{mg} / \mathrm{kg} / \mathrm{wk}$, up to a maximum of $320 \mathrm{mg}$ per week. Dose was adjusted based on weight at every NIH visit.

\section{Efficacy assessments}

Clinical daily diary symptom score. Patient signs and symptoms were recorded in standardized diaries in the English language. Each patient or caregiver was instructed to complete the diary every day for the duration of the study. The effect of each symptom (fever, joint pain, and rash) on the patient was rated for each symptom on a scale from 0 to 4 : a score of 0 indicates no symptoms, 1 indicates mild symptoms, 2 indicates moderate symptoms, 3 indicates more severe symptoms, and 4 indicates severe symptoms (possible range 0-12). Diary scores were collected for a minimum of 2 weeks prior to enrollment while patients were on anakinra. Scores were calculated as follows. (a) Average score was calculated using data entered since the previous visit, correcting for any day for which diary scores were not recorded. (b) The calculated average score for each symptom was summed and divided by the number of assessed symptoms (i.e., 3 symptoms). Diary scores were recorded on an average of $70 \%-80 \%$ of days during treatment.

Clinical dermatology score (visual analog scale). A study team member recorded dermatologic findings, including the localization of a rash, its severity, and the percentage of body surface area covered with rash. The visual analog scale ranged from 0 to $100 \mathrm{~mm}$ : $0 \mathrm{~mm}$ equals no symptoms and $100 \mathrm{~mm}$ equals severe symptoms.

Clinical joint count and quality of life questionnaires. Total joint count consisted of the assessment of 39 joints (for swelling, tenderness, redness, or range of motion). Patients or parents completed CHAQ and 
PedsQL questionnaires at baseline and at every NIH visit. The CHAQ and PedsQL questionnaires assess functional impairment and change in treatment and health-related quality of life, respectively.

Laboratory inflammatory markers. Acute-phase reactants, including ESR and CRP, were primary efficacy outcomes; white blood cell count, hemoglobin, and platelet count were also assessed at each visit.

Laboratory safety data. Complete blood cell count, with differential, serum electrolytes, renal and liver function, lipid panel, creatinine kinase, uric acid, lactate dehydrogenase, urinalysis, and other safety assessments were analyzed at the NIH clinical center laboratory. Outside laboratory tests were obtained on six occasions in five patients to follow up on a dose escalation that had previously occurred at the NIH. These laboratory values were not included in the efficacy analysis.

Laboratory rilonacept pharmacokinetic concentrations and anti-rilonacept antibodies. Trough and anti-rilonacept antibodies samples were obtained from patients at the following nominal time points: $0,1,3,4,6,12,18$, and 24 months. Blood samples were also collected 4 weeks after a dose increase. Regeneron Pharmaceutical Inc. analyzed samples for measurements of rilonacept levels in serum and anti-rilonacept antibody formation.

\section{Primary outcome}

Primary outcome is defined as the ability of rilonacept to maintain/achieve remission at 6 months, 12 months, and later 24 months. Patients were not to accrue new organ damage (mainly bone damage) or develop disease-related elevated CRP levels on rilonacept; safety was a primary endpoint. Comparisons of mean diary scores and inflammatory markers (CRP and ESR) were made between baseline and 6 months on rilonacept. All other outcomes were secondary/exploratory and included patients' questionnaires (CHAQ and PedsQL), evaluation of diary scores, acute-phase reactants at all other time points (including 3 months and 18 months), linear growth and weight assessment at each visit, and changes in bone mineral density assessed by DEXA scan betweens baseline and 12 months on rilonacept. Midparental heights and percentiles were calculated using EBMcalc (http://ebmcalc.com/HeightPotential.htm).

\section{Statistics}

DIRA is an ultra-rare disease with less than 50 patients worldwide; power calculations were not performed. Summary statistics, including means, SDs, and frequency distributions where appropriate, were calculated. Primary and secondary end point data at $3,6,12,18$, and 24 months were compared with baseline on anakinra using paired $t$ tests; all $t$ tests were 2 tailed. Comparisons were also made between data before anakinra treatment and the respective rilonacept treatment visits. One mean per patient was calculated for the available inflammatory markers from the pre-IL- 1 inhibitor treatment period and from the anakinra treatment period; these values were compared with the value obtained at the respective rilonacept visit. All $P$ values are 2 sided, except for the linear growth calculations, which are 1 sided. $P<0.05$ was considered significant. The study was designed to use two baseline values: (a) historical values for all patients that were obtained before starting anakinra (pretreatment) and (b) data from a potential anakinra withdrawal flare that might occur in the first week of switching from anakinra to rilonacept (baseline). Comparisons between anakinra and rilonacept were initially hypothesized to be not different. The absence of significant flares leading to new organ damage and the continued remission on rilonacept are considered to represent a similar degree of efficacy between anakinra and rilonacept, recognizing that formal noninferiority analysis is not possible, given the small number of patients.

\section{Study approval}

The Institutional Review Board of the NIAMS and the National Institute of Diabetes and Digestive and Kidney Diseases at the NIH approved the study. The study was conducted in accordance with the ethical principles of the Declaration of Helsinki and Good Clinical Practice guidelines. All parents or legal guardians provided informed consent. No assent was obtained from patients less than 7 years of age; all patients were younger than the assent age of 7 years at the time of enrollment. Written consent was provided for patient pictures appearing in the manuscript.

\section{Author contributions}

MG, DC, and AADJ were involved in data collection. MG and AADJ were involved in analysis of the data and the writing of the manuscript. DC, PD, RH, RRC, TLWM, AR, and JCR provided comments on the manuscript. RGM and GAMS were involved in the study design, collection and analysis of data, and the writing of the manuscript. 


\section{Acknowledgments}

The Intramural Research Program of the NIH, NIAID, and NIAMS supported this research. The authors would like to thank Sarah Benvin, Deborah Tolenaar, and Scott Mellis for their suggestions and critical review of the manuscript; Beate Stych for her support; Bahar Kost for help with data collection; and Wendy Goodspeed, Samantha Dill, and Nicole Plass for scheduling and clinical care. Last, but not least, the authors thank the patients and their families for their participation in this study.

Address correspondence to: Gina A. Montealegre Sanchez, Translational Autoinflammatory Disease Studies (TADS), National Institute of Allergy and Infectious Diseases (NIAID), Laboratory of Clinical Immunology and Microbiology (LCIM), NIH/NIAID/LCIM, Building 10, Room 11C436, 10 Center Drive, Bethesda, Maryland 20892, USA. Phone: 301.761.7747; Email: montealegrega@mail.nih.gov.

1. Sanchez GA, de Jesus AA, Goldbach-Mansky R. Monogenic autoinflammatory diseases: disorders of amplified danger sensing and cytokine dysregulation. Rheum Dis Clin North Am. 2013;39(4):701-734.

2. Aksentijevich I, et al. An autoinflammatory disease with deficiency of the interleukin-1-receptor antagonist. $N$ Engl J Med. 2009;360(23):2426-2437.

3. Reddy S, et al. An autoinflammatory disease due to homozygous deletion of the IL1RN locus. $N$ Engl J Med. 2009;360(23):2438-2444.

4. Jesus AA, et al. A novel mutation of IL1RN in the deficiency of interleukin-1 receptor antagonist syndrome: description of two unrelated cases from Brazil. Arthritis Rheum. 2011;63(12):4007-4017.

5. Brau-Javier CN, Gonzales-Chavez J, Toro JR. Chronic cutaneous pustulosis due to a 175-kb deletion on chromosome 2q13: excellent response to anakinra. Arch Dermatol. 2012;148(3):301-304.

6. Ulusoy E, Karaca NE, El-Shanti H, Kilicoglu E, Aksu G, Kutukculer N. Interleukin-1 receptor antagonist deficiency with a novel mutation; late onset and successful treatment with canakinumab: a case report. JMed Case Rep. 2015;9:145.

7. Cowen EW, Goldbach-Mansky R. DIRA, DITRA, and new insights into pathways of skin inflammation: what's in a name? Arch Dermatol. 2012;148(3):381-384.

8. Ivker RA, Grin-Jorgensen CM, Vega VK, Hoss DM, Grant-Kels JM. Infantile generalized pustular psoriasis associated with lytic lesions of the bone. Pediatr Dermatol. 1993;10(3):277-282.

9. Altiok E, et al. A novel mutation in the interleukin-1 receptor antagonist associated with intrauterine disease onset. Clin Immunol. 2012;145(1):77-81.

10. Bresnihan B, et al. Treatment of rheumatoid arthritis with recombinant human interleukin-1 receptor antagonist. Arthritis Rheum. 1998;41(12):2196-2204.

11. Bresnihan B. The prospect of treating rheumatoid arthritis with recombinant human interleukin-1 receptor antagonist. BioDrugs. 2001;15(2):87-97.

12. Mendonca LO, et al. Deficiency of interleukin-1 receptor antagonist (DIRA): report of the first Indian patient and a novel deletion affecting IL1RN. J Clin Immunol. 2017;37(5):445-451.

13. Dubois EA, Rissmann R, Cohen AF. Rilonacept and canakinumab. Br J Clin Pharmacol. 2011;71(5):639-641.

14. Economides AN, et al. Cytokine traps: multi-component, high-affinity blockers of cytokine action. Nat Med. 2003;9(1):47-52.

15. [No authors listed]. Molecule of the month. Rilonacept. Drug News Perspect. 2008;21(4):232.

16. Hoffman HM, et al. Efficacy and safety of rilonacept (interleukin-1 Trap) in patients with cryopyrin-associated periodic syndromes: results from two sequential placebo-controlled studies. Arthritis Rheum. 2008;58(8):2443-2452.

17. Hoffman HM, et al. Long-term efficacy and safety profile of rilonacept in the treatment of cryopryin-associated periodic syndromes: results of a 72-week open-label extension study. Clin Ther. 2012;34(10):2091-2103.

18. Schnellbacher C, et al. Deficiency of interleukin-1 receptor antagonist responsive to anakinra. Pediatr Dermatol. 2013;30(6):758-760.

19. Goldbach-Mansky R, et al. A pilot study to evaluate the safety and efficacy of the long-acting interleukin-1 inhibitor rilonacept (interleukin-1 Trap) in patients with familial cold autoinflammatory syndrome. Arthritis Rheum. 2008;58(8):2432-2442.

20. Ilowite NT, et al. Randomized, double-blind, placebo-controlled trial of the efficacy and safety of rilonacept in the treatment of systemic juvenile idiopathic arthritis. Arthritis Rheumatol. 2014;66(9):2570-2579.

21. Sundy JS, et al. Rilonacept for gout flare prevention in patients receiving uric acid-lowering therapy: results of RESURGE, a phase III, international safety study. J Rheumatol. 2014;41(8):1703-1711.

22. Schumacher HR Jr, et al. Rilonacept (interleukin-1 trap) for prevention of gout flares during initiation of uric acid-lowering therapy: results from a phase III randomized, double-blind, placebo-controlled, confirmatory efficacy study. Arthritis Care Res (Hoboken). 2012;64(10):1462-1470.

23. Terkeltaub RA, et al. Rilonacept in the treatment of acute gouty arthritis: a randomized, controlled clinical trial using indomethacin as the active comparator. Arthritis Res Ther. 2013;15(1):R25.

24. Di Paolo NC, Shayakhmetov DM. Interleukin $1 \alpha$ and the inflammatory process. Nat Immunol. 2016;17(8):906-913.

25. Tani-Ishii N, Tsunoda A, Teranaka T, Umemoto T. Autocrine regulation of osteoclast formation and bone resorption by IL-1 alpha and TNF- $\alpha$. J Dent Res. 1999;78(10):1617-1623.

26. Corradi A, Franzi AT, Rubartelli A. Synthesis and secretion of interleukin-1 $\alpha$ and interleukin-1 receptor antagonist during differentiation of cultured keratinocytes. Exp Cell Res. 1995;217(2):355-362.

27. Chen JD, Lapiere JC, Sauder DN, Peavey C, Woodley DT. Interleukin-1 $\alpha$ stimulates keratinocyte migration through an epidermal growth factor/transforming growth factor- $\alpha$-independent pathway. J Invest Dermatol. 1995;104(5):729-733.

28. Bando M, et al. Interleukin-1alpha regulates antimicrobial peptide expression in human keratinocytes. Immunol Cell Biol. 
2007;85(7):532-537.

29. Autmizguine J, Cohen-Wolkowiez M, Ilowite N, RAPPORT Investigators. Rilonacept pharmacokinetics in children with systemic juvenile idiopathic arthritis. J Clin Pharmacol. 2015;55(1):39-44.

30. Ferguson PJ, et al. Homozygous mutations in LPIN2 are responsible for the syndrome of chronic recurrent multifocal osteomyelitis and congenital dyserythropoietic anaemia (Majeed syndrome). J Med Genet. 2005;42(7):551-557.

31. Ferguson PJ, et al. A missense mutation in pstpip2 is associated with the murine autoinflammatory disorder chronic multifocal osteomyelitis. Bone. 2006;38(1):41-47.

32. Cox AJ, et al. Recessive coding and regulatory mutations in FBLIM1 underlie the pathogenesis of chronic recurrent multifocal osteomyelitis (CRMO). PLoS One. 2017;12(3):e0169687.

33. Minkis K, et al. Interleukin 1 receptor antagonist deficiency presenting as infantile pustulosis mimicking infantile pustular psoriasis. Arch Dermatol. 2012;148(6):747-752.

34. Stenerson M, Dufendach K, Aksentijevich I, Brady J, Austin J, Reed AM. The first reported case of compound heterozygous IL1RN mutations causing deficiency of the interleukin-1 receptor antagonist. Arthritis Rheum. 2011;63(12):4018-4022. 